\title{
Event-Triggered Multitarget Formation Control for Multiagent Systems
}

\author{
Lingmin Zhang, ${ }^{1,2}$ Xinbin Li, ${ }^{1}$ Jing Yan, ${ }^{1}$ and Xinping Guan ${ }^{3}$ \\ ${ }^{1}$ Institute of Electrical Engineering, Yanshan University, Qinhuangdao 066004, China \\ ${ }^{2}$ Institute of Mathematics and Information Technology, Hebei Normal University of Science and Technology, \\ Qinhuangdao 066004, China \\ ${ }^{3}$ School of Electronic and Electric Engineering, Shanghai Jiaotong University, Shanghai 200240, China
}

Correspondence should be addressed to Lingmin Zhang; lingmin9999@163.com

Received 23 June 2017; Revised 22 October 2017; Accepted 22 October 2017; Published 5 December 2017

Academic Editor: Leonid Shaikhet

Copyright (c) 2017 Lingmin Zhang et al. This is an open access article distributed under the Creative Commons Attribution License, which permits unrestricted use, distribution, and reproduction in any medium, provided the original work is properly cited.

The problems of multitarget selection and formation for multiagent systems are considered. First of all, an improved multitarget selection method based on auction algorithm is proposed such that each agent can automatically choose its target, and during the process of choosing targets, we apply an event-triggered mode to reduce the communication links between agents. Second, in view of the fact that all agents with the same target need to form a desired formation shape, we provide an event-triggered formation controller for each agent. Finally, we carry out the simulation experiment of the algorithm and the simulation results have illustrated the effectiveness of it.

\section{Introduction}

In recent years, the problem of multiagent system (MAS) has been widely studied by many researchers [1-4]. These systems can potentially consist of a great number of agents, such as unmanned aerial vehicles (UAG), unmanned underwater vehicles (UUV), and unmanned ground vehicles (UGA). MASs provide many applications in various practical fields, such as intelligent transportation systems, building automation, underwater exploration, and surveillance. Advantages of MASs over single agent include cost reduction, efficiency, and robustness improvement.

One interesting issue of multiagent system is formation control [5-7]. Its objective is to design algorithms to motivate agents to form a desired formation. Meanwhile, study on formation with a single target has become one of the hot spots issues in research of multiagent systems formation control [8-11]. In [8], a methodology for group coordination and cooperative control of $n$ agents to achieve a target-capturing task in 3D space was studied, and the proposed approach was based on a cyclic pursuit strategy, where agent $i$ simply pursued agent $i+1$ modulo $n$. In [9], the cooperative target pursuit problem by multiple agents based on directed acyclic graph was investigated. The target appeared at a random location and moved only when sensed by the agents, and agents pursued the target once they detected its existence. In [10], the problem of flocking motion combined with topology optimization for mobile multiagent systems was considered, and a distributed multiflocking method was designed based on the partial information exchange. In [11], the cooperative control of a team of robots to estimate the position of a moving target using onboard sensing was investigated. The above works are all based on the common assumption that a group of agents pursue the same target; that is, it is supposed that there is only one target in the workplace. However, this assumption is strict in certain situations. For instance, when more than one target is considered in the workplace, agents will face a dilemma in choosing their targets. Thus, some researchers studied multitarget formation [12-14]. In [12], a team of agents who can accomplish multitarget pursuit formation by using a developed leader-follower strategy was designed. In [13], a flocking algorithm with multitarget tracking for multiagent systems was adopted. It was supposed that each target could accept a certain number of agents. Which target would be chosen by an agent was determined by the distances from the agent to the targets. In [14], to 
solve this problem, a distributed multiflocking method was adopted based on the partial information exchange. But in the above existing multitarget selection algorithms, the targets assignment is static; that is, each agent selects an invariable target to pursue. However, as the system evolves, each agent may select a different target to pursue according to certain optimal objectives. Therefore, considering systems with dynamic targets assignment will be more significant. Thus how to design a dynamic multitarget selection is a problem to be solved.

In addition, in the existing algorithms of multitarget selection and multiagent systems formation control, timetriggered control mode is widely adopted. Time-triggered control mode is simple, but it may cause large amount of bandwidth and communication. Event-triggered control is an alternative to time-triggered control $[15,16]$. The distinct feature of event-triggered control is that control action is updated only when some specific event occurs. Compared with time-triggered control mode, event-triggered control mode has the often cited advantages on communication reduction and energy saving. It has been studied extensively in network control systems and decentralized systems $[15,17,18]$. In many cases, such as formation control, pursuit control, and path planning, it outperforms the traditional time-triggered control $[16,19-22]$. Thus, how to apply eventtriggered mode to multiagent formation control is another problem to be solved. For the above two problems, this paper focuses on the dynamic multitarget selection and formation of multiagent systems and applies event-triggered control mode to multitarget selection and formation. The main contributions of this paper are as follows:

(1) Unlike in most of the existing multitarget selection algorithms, the targets assignment is static and each agent selects an invariable target to pursue; we consider systems with dynamic targets assignment as each agent may select a different target to pursue according to certain optimal objectives. In addition, an improved dynamic selection method based on auction algorithm is adopted, and the event-triggered control mode is applied to the multitarget selection to reduce the communication links between agents.

(2) In the process of multitarget formation control for multiagent systems, we adopt the event-triggered control mode instead of time-triggered-control mode. When event-triggered control mode is applied to multiagent systems, the stability of the system can be maintained and compared with time-triggered control mode; it has the advantages of reducing the number of information updating and saving bandwidth resources and energy.

The rest of this paper is organized as follows. In Section 2, system modeling and problem formulation are presented. In Section 3, we apply the event-triggered mode to dynamic target selection and the formation strategy. Simulation studies are provided to illustrate the effectiveness of our method in Section 4. Conclusions are given in Section 5.

\section{Preliminaries and Problem Formulation}

2.1. Graph Theory. A graph $G$ is a pair that consists of a set of vertices $V=\{1,2, \ldots, N\}$ and edges $E \subseteq\{(i, j): i, j \in$ $V, j \neq i\}$. The graph is said to be undirected if $(i, j) \in$ $E \Leftrightarrow(j, i) \in E$. And in order to ensure cooperation and coordinating among agents, each agent has to know the states of other agents. Therefore, agents have to communicate with each other. Given an agent $i$, the set of agents from which it can receive information is called a neighbor set $N_{i}$; that is,

$$
N_{i}=\{j \in(1,2, \ldots, N), \quad(i, j) \in E\}, \quad i=1,2, \ldots, N .
$$

A graph is connected if any two vertices can be joined with an edge. It is assumed that the graph describing the information structure is connected. A graph also admits matrix representations. Some of these matrices, such as the adjacency matrix, the degree matrix, and the Laplacian matrix, will be reviewed subsequently.

The adjacency matrix $A(G)$ encoding of the adjacency relationship in the graph $G$ is defined as

$$
a_{i j}= \begin{cases}1, & (i, j) \in E \\ 0, & (i, j) \notin E,\end{cases}
$$

where $a_{i j}$ is the $(i, j)$ entry of the adjacency matrix $A(G) \epsilon$ $R^{n \times n}$. The degree matrix $D(G)$ for an undirected graph $G$ is a diagonal matrix $\operatorname{diag}\left\{d_{1}, d_{2}, \ldots, d_{n}\right\}$, where $d_{i}$ is the cardinality of neighbor set $N_{i}$ of agent $i$. The adjacency matrix of undirected graph is symmetric because $a_{i j}=a_{j i}$ for $i \neq$ $j$. The Laplacian matrix $L(G)$ associated with an undirected graph $G$ is defined as $L(G)=D(G)-A(G)$, where $D(G)$ and $A(G)$ are degree matrix and adjacency matrix of graph $G$, respectively.

\subsection{System Modeling and Problem Formulation}

Multiagent Systems. Define a set of agents as $\Delta=\{1,2,3, \ldots$, $N\}$, where $N$ is the number of agents. For the agent $i$ with twodimensional coordinate, the position and input vectors are denoted by $p_{i} \in R^{2}$ and $u_{i} \in R^{2}$, respectively. The dynamics of agent $i$ at time $t$ are described by the following continuoustime equation:

$$
\dot{p}_{i}(t)=u_{i} .
$$

For the dynamic system, the following assumptions are made.

Assumption 1. Initially, it is assumed that targets and agents disperse randomly in the workplace. Meanwhile, agents can detect the state information about the target at the initial time.

Assumption 2. Each agent can only obtain the state information of its neighbors and each target can only accept a certain number of agents. In the following, we will give an improved auction algorithm based on event-triggered control to complete the target selection; then an event-triggered controller for each agent will be given to form a desired formation shape. 
Input coordinate of agent $i, x_{i}, y_{i}$, coordinate of target $j, x_{j}, y_{j}$ and the cycle index $n$
(1) for $t=1: n$
(2) Calculate the distance function $L_{i j}=\sqrt{\left(x_{i}-x_{j}\right)^{2}+\left(y_{i}-y_{j}\right)^{2}}$
(3) Calculate the value of $C_{i j}=L_{i j}$
(4) for $s=1: i$
(5) Calculate the value of $v_{j}(j=1,2, \ldots, n)$
(6) Calculate $r_{i j}$ and $b_{i}$
(7) While $b_{i} \geq p$
(8) Agent $i$ will choose target $j$
(9) Inform the information to other agents
(10) $N_{j}=N_{j}-1$
(11) $p=p-0.1$
(12) end
(13) Output the position coordinate of each agent
(14) end
(15) end

Algorithm 1: Dynamic selecting algorithm based on auction.

\section{Event-Triggered Multitarget Formation}

In this section, we will design a team of agents who can accomplish multitarget formation by using an eventtriggered formation method. First, we will present an eventtriggered dynamic strategy for choosing a target. Second, we will provide an event-triggered controller for each agent to form a desired multiagent systems formation.

3.1. Strategy for Choosing a Target. In the dynamic system, each target is considered as a commodity, and we define the value of the target $i$ as $V_{i} . \forall i \neq j, V_{i}=V_{j}=V$ at the initial time, and the price to catch up with $j$ for $i$ is $C_{i j}$. The system designs an open platform, in which all the $m$ targets have an auction, and all the $N$ agents are involved in the auction. In this mode of auction, the auction platform begins with a given price, and all agents are aware of the current price announced. The price is gradually reduced, until some agent selects it. In the designed auction algorithm, all targets are simultaneously on auction. When an agent's income is greater than or equal to the outcry of the current system, the agent selects the corresponding target. If target $j$ is chosen by $N_{j}$ agents, the value obtained by agent $i$ is

$$
V_{i}=\frac{V}{N_{j}} .
$$

When $N_{j}$ increases, the value obtained from target $j$ will be decreased gradually, and thus the agent will tend to choose the target that is chosen by fewer agents. In this way, we can effectively avoid the problem of selecting the same target for many agents.

Building the proceeds functions as follows:

$$
r_{i j}=V_{j}-C_{i j}
$$

where $C_{i j}=L_{i j}$ is the cost function for agent $i$ to select target $j, L_{i j}=\left\|P_{i}-e_{j}\right\|$ and $P_{i}$ and $e_{j}$ are positions of agent $i$ and target $j$, respectively. After calculating its proceeds for selecting each target, the agent will choose the one with the biggest proceeds as its target. In this way, each agent will tend to choose target relatively close to it, in order to get the final rate of exchange:

$$
b_{i}=\max \left\{r_{i 1}, r_{i 2}, \ldots, r_{i n}\right\} .
$$

When the current bid price $p$ is less than $b_{i}$ or equal to $b_{i}$, agent $i$ will select the corresponding target and drop out of the auction. The remaining agents will continue to achieve the selection until the last agent accomplishes the target selection. After selecting the targets, agents will continue to move and will update the data at the next time node, in order to achieve the dynamic selection and make a response to the changes of the scene. The pseudocode of the auction algorithm is showed in Algorithm 1.

In the design of the algorithm, if the $j$ th target has been selected, $N_{j}=N_{j}+1$, the $i$ th agent may select the further target. In the next choice, as the agent's position has been changed and the value of $N_{j}$ may have been changed, agent $i$ may select more nearer target, and then the target replacement phenomenon occurs. In addition, as the time-triggered control mode is adopted in the multitarget dynamic selection, the agent will update the selection data at each sampling time and make a target selection. It will lead to frequent calculation of the agent's own proceeds, frequent replacement of the target, and large amount of communication among agents. In addition, it takes up a lot of bandwidth and will result in a lot of unnecessary energy consumption. For solving the above problems, in the improved algorithm, we define the target value as follows:

$$
V_{i j}=V+\varepsilon \quad(\varepsilon>0)
$$

where $V$ is the initial value of the target in the system and $\varepsilon$ is the value increment of target $j$ relative to agent $i$. In this way, when the target proceeds $V_{i j}$ are changed, the gain of the agent selecting the original target will be increased, and thus 
it will tend to select the target selected last time. In addition, we introduce an event-triggered function [22]

$$
\left\|e_{i}\left(t_{k}+l h\right)\right\|_{2}^{2} \leq\left\|z_{i}\left(t_{k}+l h\right)\right\|_{2}^{2},
$$

where $h$ is the sampling period for all agents synchronized by a clock, $e_{i}\left(t_{k}+l h\right)$ is defined as the position difference at the last event time and the currently sampled time

$$
e_{i}\left(t_{k}+l h\right)=x_{i}\left(t_{k}\right)-x_{i}\left(t_{k}+l h\right),
$$

and $z_{i}\left(t_{k}+l h\right)$ is the comparison of the position of agent $i$ with all its neighbors

$$
z_{i}\left(t_{k}+l h\right)=\sum x_{i}\left(t_{k}\right)-x_{j}\left(t_{k}+l h\right) .
$$

At each sampling instant, each agent broadcasts its state information to the neighbors and also receives state information from its neighbors for event detection. If the condition in (8) is satisfied, the agent remains selecting the same target; otherwise, a new round of target selection will be carried out. This process is defined as an event trigger, and the system updates the input information and the deviation value $e_{i}\left(t_{k}+l h\right)$, to continue to begin the next triggering. By adding an increment $\varepsilon$ in the value function, the agents can keep selecting the fixed target, and when the number of the targets is changed in the scene, they will response to the changing and change the target in time.

Corollary 3. When $V=4 L_{\max }+\alpha$, where $V$ is the initial value of each agent, $L_{\max }$ is the maximum of $L_{i j}$, and $0<\varepsilon<V / 2$, the agent will respond to the state changing of the scene in the dynamic selection and will not be affected by the increment.

Proof (proof by contradiction). Suppose that there appears a new target $t$ in the system and the number of targets is less than that of the agents; the new target $t$ can not be selected by any agent. Since the new target is not chosen by any agent, $r_{i t}<r_{\min }$, where $r_{\min }$ is the minimal proceeds of $i$ and the new target $t$ is given the initial value $V$. As the target has not been selected by any agent, $N_{t}=1$, then the agent calculates the proceeds of choosing target $t$. And

$$
r_{\min }<\frac{V+\varepsilon_{\max }}{N_{j}}-L_{i j}=\frac{3 V}{2 N_{j}}-L_{i j}, \quad N_{j} \geq 2 .
$$

Thus $r_{\min }<3 V / 4$.

And because $r_{i t}=V-L_{i t} \geq V-L_{\max } \geq 3 V / 4$, we can obtain that $r_{i t}>r_{\min }$.

\subsection{The Event-Triggered Multitarget Formation}

Remark 4. In this paper, there are $N$ agents and $m$ targets. Namely, $N$ agents will be divided into $m$ groups based on the strategy of choosing targets. Agents with the same target will be in the same group. For simplicity, we only provide the control method to one group of agents. The proposed method can then be extended to the remaining $m-1$ groups by updating the number of agents in each group. In the specified group, $n(n<N)$ agents are considered

$$
\dot{x}_{i}(t)=u_{i}, \quad i=1,2, \ldots, n,
$$

where $x_{i}$ and $u_{i}$ are state and controlled input of agent $i$, respectively. Then the governing equations can be described as

$$
u_{i}(t)=-\sum_{j \in N_{i}} a_{i j}\left(x_{i}(t)-x_{j}(t)\right),
$$

where $a_{i j}$ is the $(i, j)$ entry of the adjacency matrix, $N_{i}$ is the neighbor set of agent $i$ at time $t$, and $u_{i}$ is the control input. In this design, the control equation of (13) can be changed to

$$
u_{i}(t)=-\sum_{j \in N_{i}} \phi\left(x_{i}(t)-x_{j}(t)\right)
$$

where $\phi=a_{i j}$. And the formation control method adopted in this design is the one with an offset. As the formation control method adopts a static formation keeping mode, the deviation is a constant. The state with offset is

$$
\widehat{x}_{i}(t)=x_{i}-\gamma_{i}
$$

As $\gamma_{i}$ is a constant, we can obtain that

$$
\dot{\hat{x}}_{i}(t)=\dot{x}_{i}
$$

The equation with offset can also be applied to the control equation of (14), and then the equation with offset is

$$
u_{i}(t)=\sum_{j \in N_{i}} \phi\left(\widehat{x}_{i}(t)-\widehat{x}_{j}(t)\right) .
$$

By applying the control equation, the neighboring agents of the system can be given the desired formation. In the formation problem, the essence of event-triggered control mode is to propose an event-triggered control mechanism, in order to reduce the communication between neighboring agents and the energy consumption of event detection for each agent. The event condition for agent $i$ has the form of (8). The event-triggered control method is applied to the agents' formation control with an offset, and then (17) is as follows:

$$
\dot{\hat{x}}_{i}(t)=\sum_{j \in N_{i}} \widehat{x}_{i}\left(t_{k}\right)-\widehat{x}_{j}\left(t_{k}\right) \text {. }
$$

When the deviation $e_{i}\left(t_{k}+l h\right)$ in (9) is added to (18)

$$
\begin{aligned}
\dot{\hat{x}}_{i}(t)= & \sum_{j \in N_{i}} \widehat{x}_{i}\left(t_{k}\right)-\widehat{x}_{j}\left(t_{k}\right) \\
= & -\sum_{j \in N_{i}} \widehat{x}_{i}\left(t_{k}+l h\right)-\widehat{x}_{j}\left(t_{k}+l h\right) \\
& -\sum_{j \in N_{i}} \widehat{x}_{i}\left(t_{k}\right)-\widehat{x}_{i}\left(t_{k}+l h\right) \\
& +\sum_{j \in N_{i}} \widehat{x}_{j}\left(t_{k}\right)-\widehat{x}_{j}\left(t_{k}+l h\right) .
\end{aligned}
$$

By [22], we know that when the event-triggered control mode is applied to multiagent systems, the stability of the system can be maintained, and compared with time-triggered control mode, the event-triggered control mode has the advantage of saving energy. 
TABLE 1: Positions of the agents and targets.

\begin{tabular}{lccccccccc}
\hline & Agent 1 & Agent 2 & Agent 3 & Agent 4 & Agent 5 & Agent 6 & Target 1 & Target 2 & Target 3 \\
\hline$X(\mathrm{~m})$ & 0.4 & 0.4 & 0.9 & 0.9 & 1.4 & 1.4 & 9.4 & 4.8 \\
$Y(\mathrm{~m})$ & 0.4 & 1.4 & 0.1 & 2.0 & 0.4 & 1.4 & 3.1 & 9.7 & 8.7 \\
\hline
\end{tabular}

TABLE 2: Final results of the targets allocation in Figure 1(d).

\begin{tabular}{llllllr}
\hline & Agent 1 & Agent 2 & Agent 3 & Agent 4 & Agent 5 & Agent 6 \\
\hline $0<t<200$ & Target 1 & Target 2 & Target 1 & Target 2 & Target 2 \\
$t \geq 200$ & Target 3 & Target 2 & Target 1 & Target 3 & Target 2 & Target 1 \\
\hline
\end{tabular}

TABLE 3: The original positions of the agents.

\begin{tabular}{lcccc}
\hline & $X$ coordinate $(\mathrm{m})$ & $X$ offset $(\mathrm{m})$ & $Y$ coordinate $(\mathrm{m})$ & $Y$ offset $(\mathrm{m})$ \\
\hline Agent 1 & 3.23 & 0 & 4.61 & 0.5 \\
Agent 2 & 5.67 & 0 & 2.31 & -0.5 \\
Agent 3 & 9.81 & 0.5 & 1.11 & 0 \\
\hline
\end{tabular}

\section{Simulation Results}

This section presents the simulation of the proposed multitargets selection and formation method. Initially, the agents and targets are randomly dispersed in the workplace and the scene size is $10 \times 10$.

4.1. Simulation of the Target Selection. The original value of the target is $V=56$, and there are 6 agents and 2 targets (Target 1 and Target 2). In Figure 1(d), when $t=200$, there appears a new target (Target 3 ). The initial positions of the agents and targets are in Table 1.

Comparing Figure 1(a) with 1(b), it can be seen that when applying the improved algorithm in the target selection, the agents do not exchange the target frequently, and the agents do not appear to get together to choose the same target. In addition, comparing Figure 1(b) with 1(c), it can be seen that when applying the event-triggered control mode instead of time-triggered control mode, we can not only obtain the expected results but also reduce communication among agents, thus reducing the energy consumption.

Based on Table 2, we can see that when the system increases a new target, two agents abandon the original target and choose the new one. It can be seen that the incremental value $\varepsilon$ does not affect the response of the system to the changing of the target number.

\subsection{Simulation of the Event-Triggered Formation Control.} Based on Figures 1(a), 1(b), 1(c), and 1(d), we can see that state of the agents tend to be consistent due to presence of the offset, and the input value tends to be zero. Thus we know that, by applying offset in the formation, the multiagent system can form a desired formation.

Furthermore, we choose agents with the original positions as in Table 3. Based on Figures 1(e)-1(k), we can see that when applying event-triggered control mode in the formation control, the result of the system basically has no difference with that of the time-triggered control mode, and thus the feasibility of event-triggered control mode in the formation control is verified. Meanwhile, it can reduce the times of updating information and lengthen the interval time of updating and thus can reduce the consumption of resources and energy greatly.

The essence of event-triggered control is to control the event condition, so the deviation $e$ and event-triggered value $z$ are used to define the event occurring. Based on Figure 1(1), we can see that when the system is running, the amount of deviation $e$ is greater than that of the comparison value $z$.

\section{Conclusions}

The multitarget dynamic selection method and the eventtriggered formation control strategy of multiagent systems are presented in this paper. First, as in most of the existing multitarget selection algorithms, the targets assignment is static and each agent selects an invariable target to pursue; however, in many practical application, as the system evolves, each agent may select a different target to pursue. Thus we give the dynamic multitarget selection algorithm based on auction to solve this problem and apply event-triggered control mode to it. Second, as the event-triggered control mode has the advantages of reducing the number of information updating and saving bandwidth resources and energy, while giving the formation of agents that choose the same target, in the formation process, the event-triggered control mode is adopted instead of the time-triggered control mode.

In our future work, for taking full advantages of the eventtriggered control mode, we will apply it to more multiagent formation problems.

\section{Conflicts of Interest}

The authors declare that they have no conflicts of interest.

\section{Authors' Contributions}

Lingmin Zhang and Xinbin Li carried out the proof of the theorems and gave the simulation, and Jing Yan and Xinping 


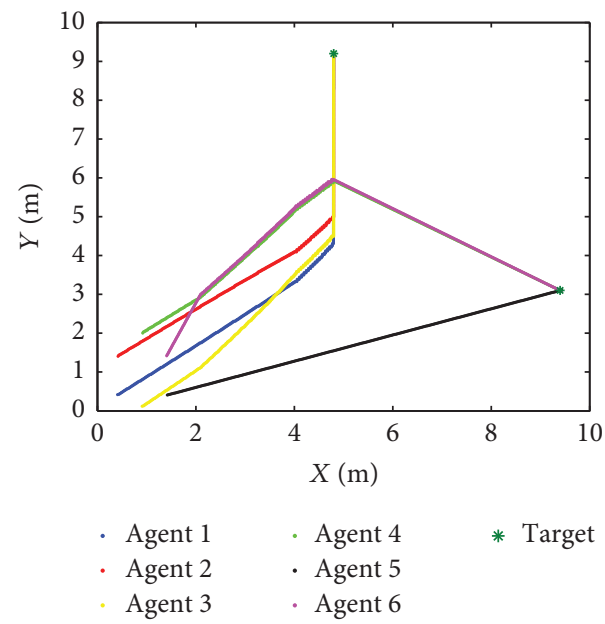

(a)
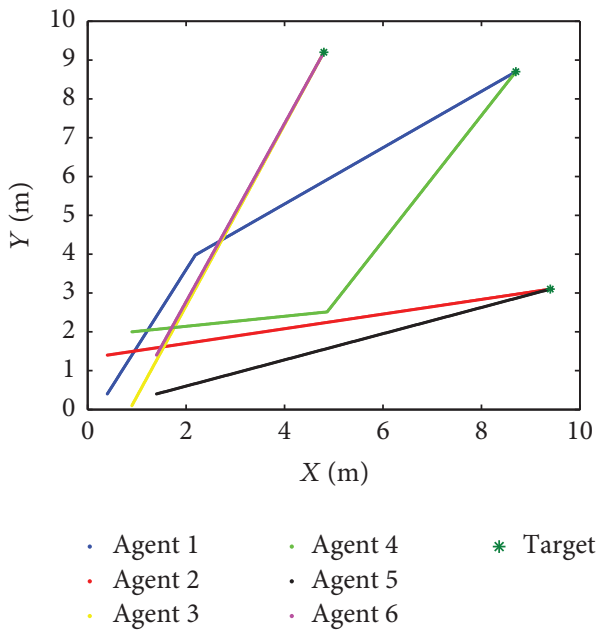

(c)

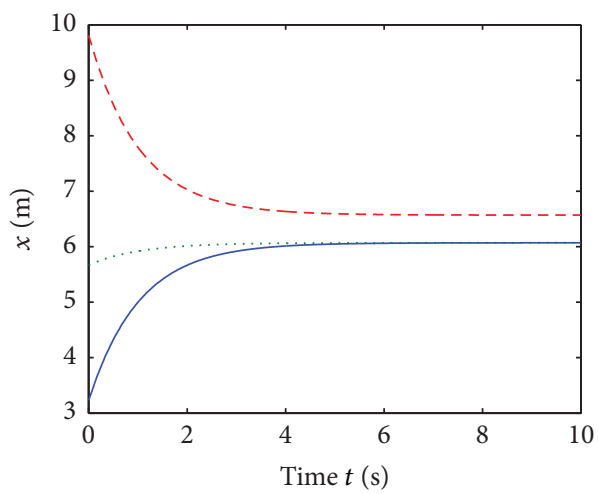

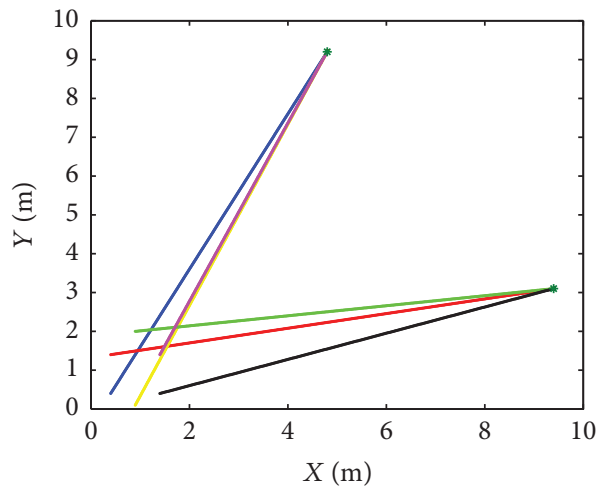

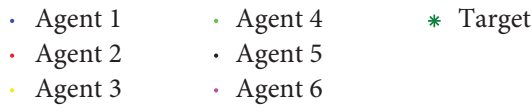

(b)
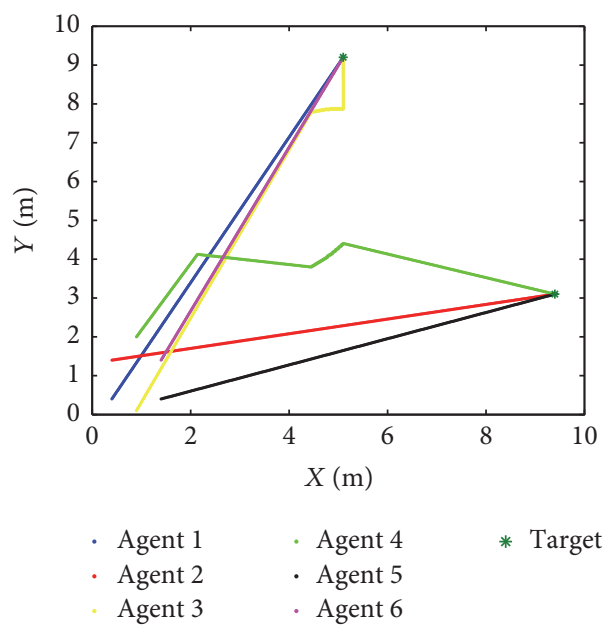

(d)

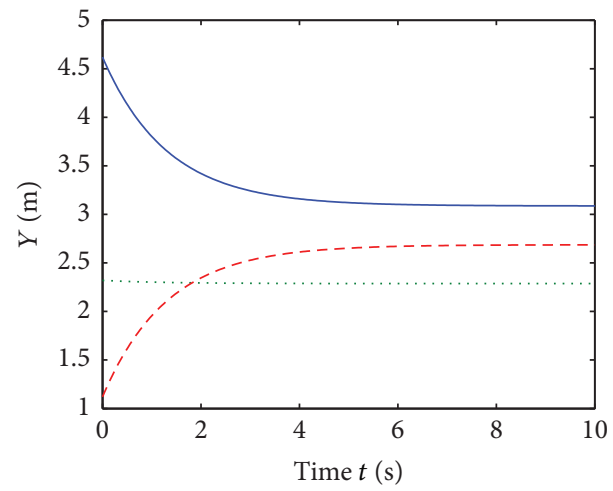

$-y_{1}(t)$

…. $y_{2}(t)$

- - $y_{3}(t)$

(e)

(f)

FIgure 1: Continued. 

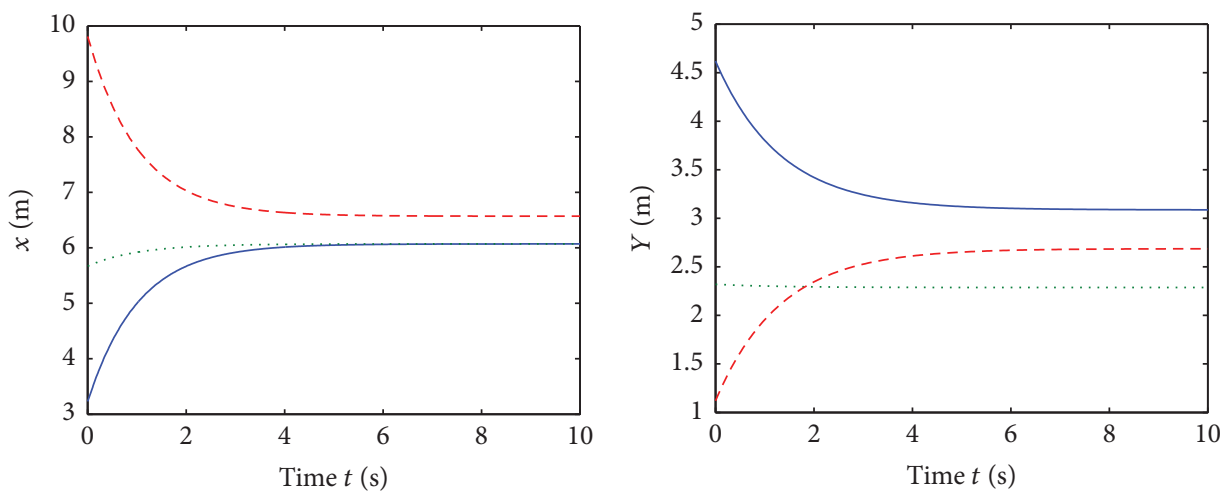

$$
\begin{array}{ll}
- & x_{1}(t) \\
\ldots \ldots & x_{2}(t) \\
--- & x_{3}(t)
\end{array}
$$

(g)

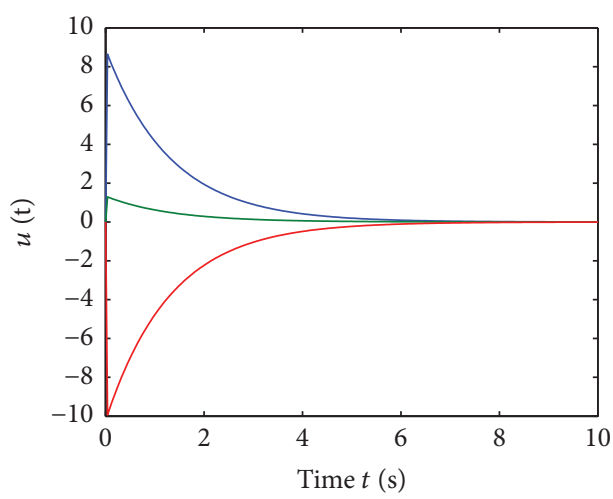

$$
\begin{aligned}
& -u_{1}(t) \\
& -u_{2}(t) \\
& -u_{3}(t)
\end{aligned}
$$

(i)

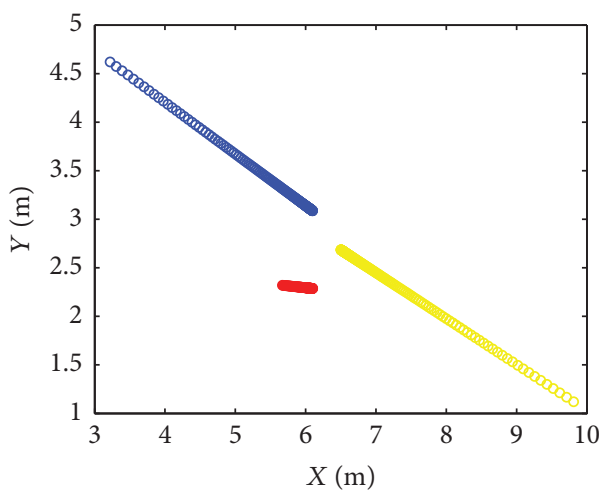

$$
\begin{aligned}
& \text { - Target } 1 \\
& \text { - Target } 2 \\
& \text { - Target } 3
\end{aligned}
$$

(k)

$$
\begin{array}{ll}
- & y_{1}(t) \\
\ldots \ldots & y_{2}(t) \\
--- & y_{3}(t)
\end{array}
$$

(h)

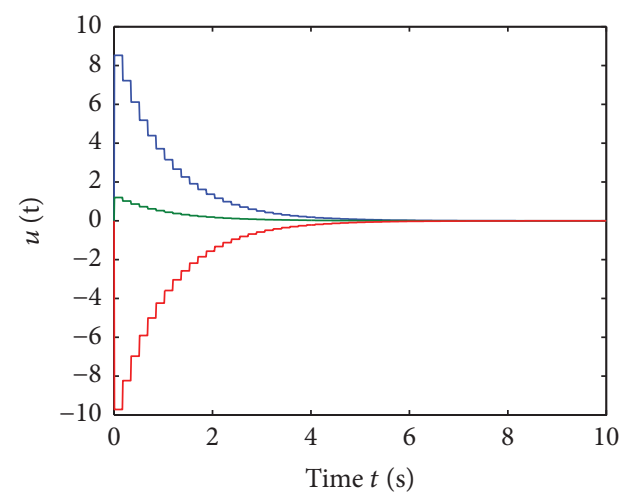

$$
\begin{aligned}
& -u_{1}(t) \\
& -u_{2}(t) \\
& -u_{3}(t)
\end{aligned}
$$

(j)

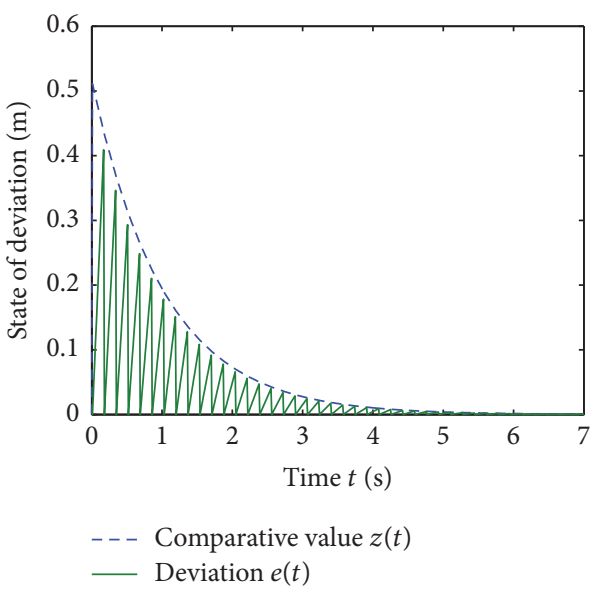

(l)

FIGURE 1: (a) Selecting result of the original algorithm. (b) Selecting result of the improved algorithm with an increment $\varepsilon$. (c) Selecting result of the improved algorithm based on event-triggered mode. (d) Response of the system when increasing a new target. (e) $X$ under timetriggered control model. (f) $Y$ under time-triggered control model. (g) $X$ under event-triggered control model. (h) $Y$ under event-triggered control model. (i) Input under time-triggered control model. (j) Input under event-triggered control model. (k) Construction of formation control. (l) Deviation value $\varepsilon$ and event-triggered value $z$. 
Guan carried out the check of the manuscript. All authors read and approved the final manuscript.

\section{Acknowledgments}

The work was partially supported by NSF of China under 61503320, 61571387, and 61172095, by China Postdoctoral Science Foundation Funded Project under 2015M570235, by Youth Foundation of Hebei Educational Committee under QN2015187, by Postdoctoral Science Foundation Funded Project of Hebei Province under B2015003018, by the Open Project Program of Key Laboratory of System Control and Information Processing, Ministry of Education, under Scip201501, and by the Self-Determined Project of Yanshan University under 14LGA010 and 14LGA020.

\section{References}

[1] G. Antonelli, F. Arrichiello, and S. Chiaverini, "Flocking for multi-robot systems via the Null-space-based behavioral control," Swarm Intelligence, vol. 4, no. 1, pp. 37-56, 2010.

[2] Y. Liu and Y. Jia, "An iterative learning approach to formation control of multi-agent systems," Systems \& Control Letters, vol. 61, no. 1, pp. 148-154, 2012.

[3] Y. Zhao, Z. Duan, G. Wen, and G. Chen, "Distributed finite-time tracking for a multi-agent system under a leader with bounded unknown acceleration," Systems \& Control Letters, vol. 81, pp. 8-13, 2015.

[4] Y. Dai, Y. Kim, S. Wee, D. Lee, and S. Lee, "A switching formation strategy for obstacle avoidance of a multi-robot system based on robot priority model," ISA Transactions ${ }^{\circledR}$, vol. 56, pp. 123-134, 2015.

[5] J.-L. Wang and H.-N. Wu, "Leader-following formation control of multi-agent systems under fixed and switching topologies," International Journal of Control, vol. 85, no. 6, pp. 695-705, 2012.

[6] X. Lu, F. Austin, and S. Chen, "Formation control for secondorder multi-agent systems with time-varying delays under directed topology," Communications in Nonlinear Science and Numerical Simulation, vol. 17, no. 3, pp. 1382-1391, 2012.

[7] X. Chen and F. Hao, "Event-triggered average consensus control for discrete-time multi-agent systems," IET Control Theory \& Applications, vol. 6, no. 16, pp. 2493-2498, 2012.

[8] T.-H. Kim and T. Sugie, "Cooperative control for targetcapturing task based on a cyclic pursuit strategy," Automatica, vol. 43, no. 8, pp. 1426-1431, 2007.

[9] J. Yan, X.-P. Guan, and X.-Y. Luo, "Consensus pursuit of heterogeneous multi-agent systems under a directed acyclic graph," Chinese Physics B, vol. 20, no. 4, Article ID 048901, 2011.

[10] X. Luo, D. Liu, X. Guan, and S. Li, "Flocking in target pursuit for multi-agent systems with partial informed agents," IET Control Theory \& Applications, vol. 6, no. 4, pp. 560-569, 2012.

[11] K. Hausman, J. Müller, A. Hariharan, N. Ayanian, and G. S. Sukhatme, "Cooperative control for target tracking with onboard sensing," International Symposium on Experimental Robotics, 2014.

[12] J. Yan, X.-P. Guan, and X.-Y. Luo, "Multi-target pursuit formation of multi-agent systems," Chinese Physics B, vol. 20, no. 1, Article ID 018901, 2011.

[13] X. Luo, S. Li, and X. Guan, "Flocking algorithm with multitarget tracking for multi-agent systems," Pattern Recognition Letters, vol. 31, no. 9, pp. 800-805, 2010.
[14] H. Pei, S. Chen, and Q. Lai, "Multi-target consensus circle pursuit for multi-agent systems via a distributed multi-flocking method," International Journal of Systems Science, vol. 47, no. 16, pp. 3741-3748, 2016.

[15] T. Henningsson, E. Johannesson, and A. Cervin, "Sporadic event-based control of first-order linear stochastic systems," Automatica, vol. 44, no. 11, pp. 2890-2895, 2008.

[16] J. Lunze and D. Lehmann, "A state-feedback approach to eventbased control," Automatica, vol. 46, no. 1, pp. 211-215, 2010.

[17] J. Mazo and P. Tabuada, "Decentralized event-triggered control over wireless sensor/actuator networks," Institute of Electrical and Electronics Engineers Transactions on Automatic Control, vol. 56, no. 10, pp. 2456-2461, 2011.

[18] M. Mazo and M. Cao, "Asynchronous decentralized eventtriggered control," Automatica, vol. 50, no. 12, pp. 3197-3203, 2014.

[19] D. Xie, S. Xu, Y. Chu, and Y. Zou, "Event-triggered average consensus for multi-agent systems with nonlinear dynamics and switching topology," Journal of The Franklin Institute, vol. 352, no. 3, pp. 1080-1098, 2015.

[20] H. Zhang, R. Yang, H. Yan, and Q. Chen, "Distributed eventtriggered control for consensus of multi-agent systems," Journal of The Franklin Institute, vol. 352, no. 9, pp. 3476-3488, 2015.

[21] B. Watkins, S. Al-Areqi, S. Reimann, and S. Liu, "Event-based control of constrained discrete-time linear systems with guaranteed performance," International Journal of Sensors Wireless Communications and Control, vol. 5, no. 2, pp. 72-80, 2015.

[22] X. Meng and T. Chen, "Event based agreement protocols for multi-agent networks," Automatica, vol. 49, no. 7, pp. 2125-2132, 2013. 


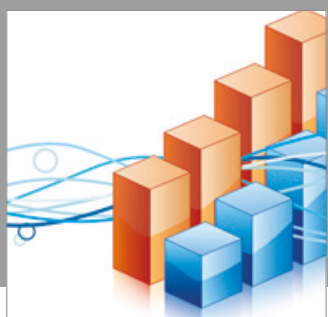

Advances in

Operations Research

vatersals

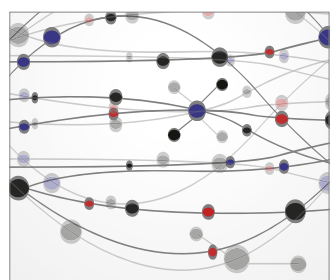

\section{The Scientific} World Journal
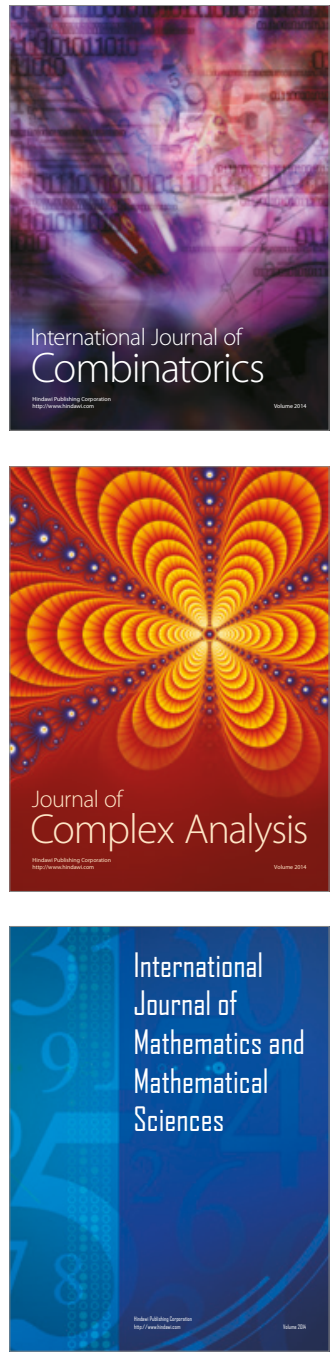
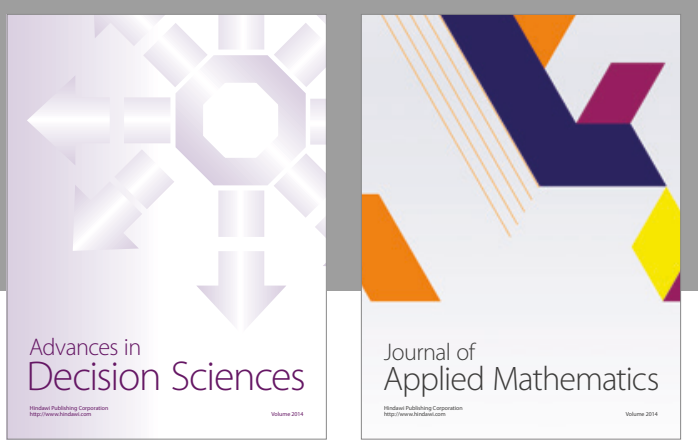

Algebra

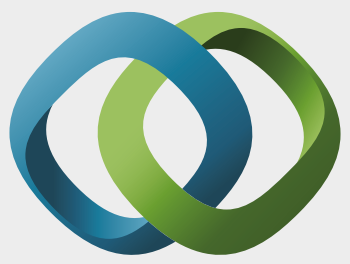

\section{Hindawi}

Submit your manuscripts at

https://www.hindawi.com
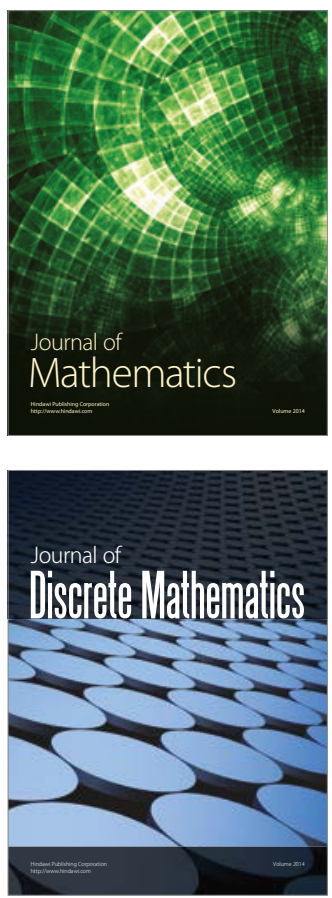

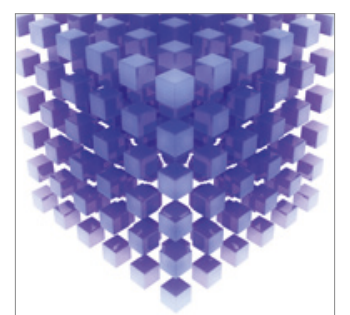

Mathematical Problems in Engineering
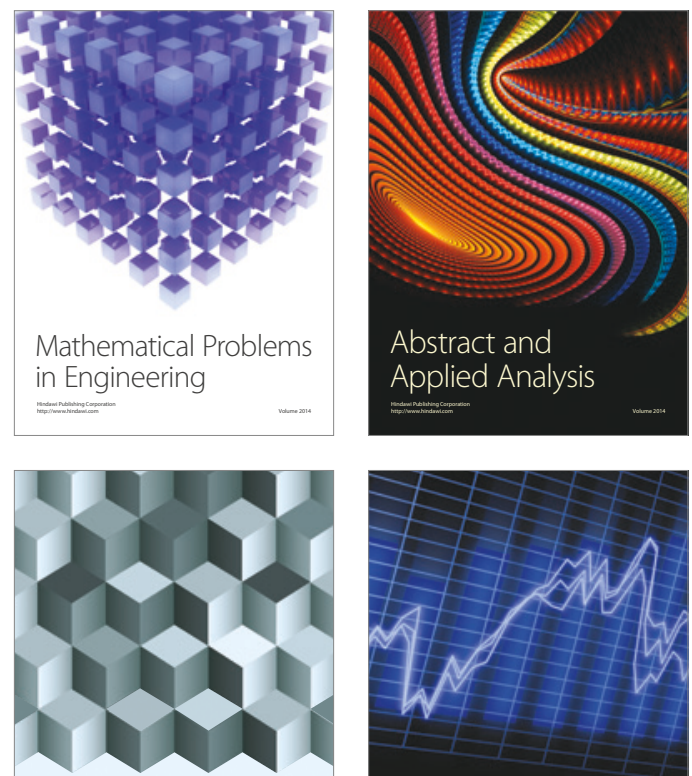

Journal of

Function Spaces

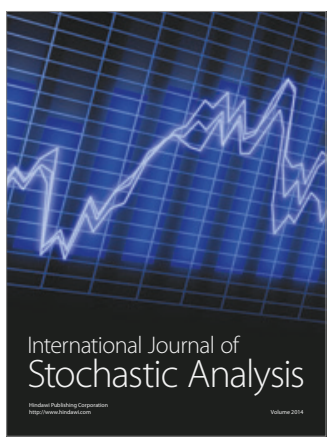

Probability and Statistics
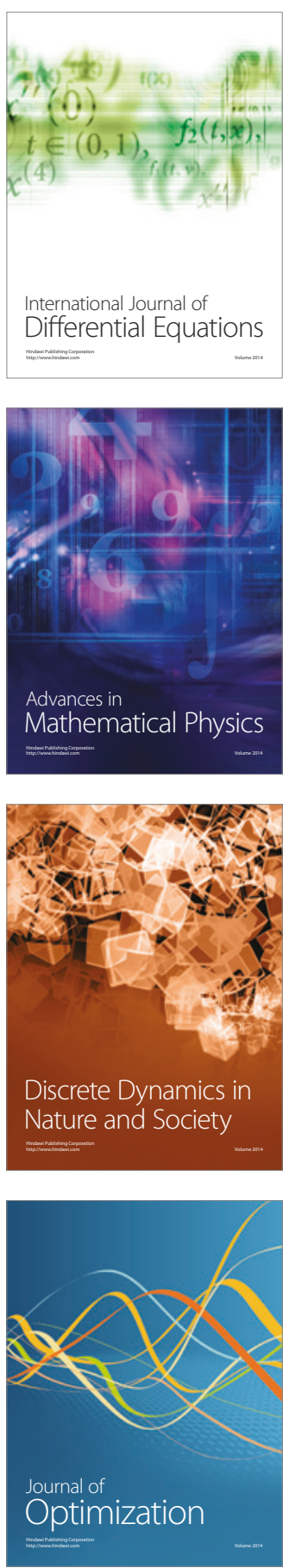